PROTOZOOLOGIC AND CLINICAL STUDIES ON THE

TREATMENT OF PROTOZOAL DYSENTERY

WITH BENZYL BENZOATE

I. A PRELIMINARY REPORT ON EIGHT CASES OF ENDAMEBIC DYSENTERY

AND ONE CASE OF BACILLARY DYSENTERY TREATED AT

THE PHILIPPINE GENERAL HOSPITAL

FRANK G. HAUGHWOUT, M.D., AND PEDRO T. LANTIN, M.D.

MANILA, P. I.

WITH AN ADDENDUN BY

M. A. ASUZANO, M.D.

BAGLIO, P. I.

Several months ago the attention of the senior author was attracted by the investigation of the action of the benzyl esters carried out by Macht ${ }^{1}$ at Johns Hopkins University. The pharmacologic action of the two esters, benzyl acetate and benzyl benzoate, which formed the subject of Macht's investigations, suggested, as Macht has pointed out, their application to the treatment of the protozoal dysenteries, especially in view of the nontoxic nature of these infections. In his paper, Macht cited a case in which benzyl benzoate was applied, with apparent success, in the treatment of a case of endamebiasis of fifteen years' standing which had been contracted in the Philippine Islands, and this determined us to make a trial of the drug in the wards of the Philippine General Hospital.

Some benzyl benzoate was prepared locally for us and it was found to exert no untoward effects on guinea-pigs when injected pure in moderately heavy doses; but when it was tried out on one or two patients it was fotnd to contain some traces of an impurity which set up a slight degree of gastric irritation and we decided to discontinue its use. Later, we used some of a small batch of the drug prepared at the Bureau of Science, Manila, in three cases. One patient of the three complained of slight nausea; the others took it without experiencing any ill effects.

The senior author entered into correspondence with Dr. Macht, who very kindly had the manufacturers, Messrs Hynson, Westcott \& Dunning, of Baltimore, send us a small supply of the pharmaceutically prepared miscible benzyl benzoate, put up in 20 per cent. alcoholic solution. This was used in all of the cases hereinafter recorded except

1. Macht, David I.: A Pharmacological and Therapeutic Study of Some Benzyl Esters, J. Pharmacol \& Exper. Therap. 11:419 (July) 1918. 
in the three already mentioned, and in no instance did we observe any untoward effects on the gastrointestinal or urinary tracts, or, indeed, ill effects of any nature.

In starting the work it must be confessed that we had to cope with the prevailing opinion held by many tropical workers, that it is unwise to check the diarrhea in dysentery except through the medium of the specific treatment employed. We are inclined to disapprove of the administration of bismuth or opium in such cases, but we think the objection lies mainly in the treatment of bacillary dysentery which we have only briefly touched on in this series. The protozoal dysenteries are nontoxic, that is to say, so far as the protozoal infection is concerned, and we consider that there is much less danger in checking the diarrhea caused by them than is to be feared from checking the diarrhea of bacillary dysentery, especially through the use of opium. The checking of the diarrhea through the slowing down of peristalsis has been a striking feature of the results we have observed in this series, and in no case has this action been attended by other than good results.

It is our intention to continue these studies. We shall not limit our work to the treatment of endamebiasis, but, as opportunity offers, shall try it in the treatment of the dysenteries caused by Balantidium colt and the flagellated protozoa found in the human intestinal tract. The supply of the drug obtained through Dr. Macht was limited, and much of it was saved for use in the treatment of a case of lymphatic leukemia by the same author, in collaboration with Dr. Asuzano, the results of which will be published elsewhere. Our principal object in connection with this short series, was to accustom ourselves to the action of the drug and to outline plans for a more systematic study of its action in the future. In our next paper we shall give a fuller treatment of the action of the drug on the parasites. This paper is mainly a clinical study.

Ten cases were selected in the wards of the hospital and subjected to the treatment to be described. Of these cases, eight were endamebic uncomplicated by infection with Bacillus dysenteria, one was bacteriologically positive for Bacillus dy'senteriae (Shiga) and showed no evidence of coexisting endamebic infection. The other case was discovered to be one of miliary tuberculosis, in a late stage, with diarrhea. The patient was dropped from the series and died a few days later. To these must be added two cases of endamebiasis treated at the Baguio Hospital by Dr. Asuzano, whose report appears as an addendum to this paper. All the patients left the hospital either free from dysenteric symptoms of any kind, or so markedly improved that they refused to stay longer. How many of these cases represent cures, in 
the generally accepted sense, can only be determined by future developments. In no case, except in one of Dr. Asuzano's cases (Case 2), has it been possible to keep the patient under observation. We are reluctant to pronounce a case of endamebiasis "cured" until the stools have been free from trophozoites or cysts for a period of at least thirty days. We incline to the opinion that this period is none too long. Endamebiasis is essentially a chronic disease, as is malaria, and is prone to exhibit surprises to the clinician and microscopist, not dissimilar to syphilis.

Another factor that must be borne in mind in the event that any of these patients applies in the future for treatment of endamebiasis, is that all of them, with the exception of Dr. Asuzano's cases, were individuals belonging to the poorer classes whose ideas of hygiene and sanitation are not even elementary. Unless cases can be controlled with almost military rigor, as may sometimes be done with the inmates of a prison, the worker in the tropics is always uncertain in such cases whether he is dealing with a recrudescence of the original attack or a fresh infection.

In each case we made every effort to secure a correct diagnosis, and we believe that we have been successful in every one of the cases we are presenting. The microscope was used in the determination of the type or types of parasites present and to discover the nature of the cellular exudate carried in the stool. This was checked up, in each instance, where there was the slightest suspicion of a coexisting bacillary infection, by bacteriologic examination by Dr. Walfrido de Leon of the department of pathology and bacteriology, to whom the authors extend their thanks. The customary weight was also placed on the clinical manifestations.

Microscopic diagnosis réquires careful interpretation by an experienced worker, but we believe that, in the main, it is more to be relied on than bacteriologic diagnosis under the methods and technic now arailable to the bacteriologist. We base a diagnosis of endamebiasis only on the finding of the trophozoites of Endameba dysenterica as shown by amebae containing ingested erythrocytes, or the discovery of the characteristc quadrinucleated cysts. The character of the exudate carries much weight with us, however. If the cellular elements of the stool are scanty in amount, contain few leukocytes of the polymorphonuclear type and exhibit cells that show evidence of having undergone proteolytic digestion, we suspect endamebiasis, but suspend judgment until we have identified the ameba.

We have found the bacteriologic examination of stools for Bacillus dysenteriae a weak reed to lean on. A negative report carries no weight with us. It is our practice to insist that the stool be carried to the 
bacteriologist immediately after it has been passed, for experience has shown us, as it has shown many other workers, that delay in the examination of the stools lessens the chances of detecting the bacillus.

The character of the exudate is of value as has been shown by Wenyon and O'Connor, ${ }^{2}$ Willmore and Shearman ${ }^{3}$ and others, although many of the distinctive features they have pointed out have been known to tropical workers for a long time. We regard as suspicious of bacillary dysentery a stool that is rich in cellular exudate, mostly of a polymorphonuclear nature, one that is strongly indicative of an acute inflammatory process and of toxic necrosis.

Stools of the latter type are often found to contain Endameba dysenterica. In such cases it is customary to regard the condition as probably one of mixed bacillary and endamebic infection. Infections of this type are common in the Philippine Islands. But, as time goes on, the conviction is growing on us that such stools are not necessarily indicative of mixed bacillary and endamebic dysentery. We are not in entire accord with authors who hold that endamebic dysentery does not become complicated by invasion of the amebic ulcers in the intestinal wall by bacteria other than Bacillus dysenteriae with consequent evidence, in the stools, of an acute inflammatory process, and we lean strongly towards the statement made by Cowan and Miller ${ }^{4}$ that they doubt the existence of pure endamebic infection as they are skeptical of the existence of chronic bacillary dysentery. It is this factor that seems to us to inject an element of doubt into the otherwise apparently reliable method of cytodiagnosis as employed in the dysenteries.

In addition to the direct examination of samples taken from different parts of the stools, it is our practice to concentrate the stools after a modification of the method of Cropper and Row." This is done when the patient is admitted to determine the extent of associated parasitism present and again when the patient is discharged from the hospital in order to detect the cysts of Endameba dysenterica. Table 1 shows the result of the examination in each case on admission.

As a routine measure, all our patients received aperient sulphates. In some instances enemas of potassium permanganate were given to clear out the bowel when the stools were formed. Ipecacuanha, when

2. Wenyon, C. M., and O'Conner, F. W.: An Inquiry Into Some Problems Affecting the Spread and Incidence of Protozoal Infections of British Troops and Natives in Egypt, etc., J. Roy. Army Med. Corps 28:151, 1917.

3. Willmore, J. Graham, and Shearman, Cyril H.: On the Differential Diagnosis of the Dysenteries, Lancet 2:200 (Aug. 17) 1918.

4. Cowan, John M., and Miller. Hugh: Dysentery: a Clinical Study, J. Roy. Army Med. Corps 31:277, 295, 1918.

5. Cropper, J. W., and Row, R. W. Harold: A Method of Concentrating Endamoeba Cysts in Stools, Lancet 1:179 (Feb. 3) 1917. 
administered, was given in the form of salol coated pills. The dose on each of three successive nights was $180 \mathrm{cg}$. In acute cases we give $4 \mathrm{cg}$. of emetin hydrochlorid daily hypodermically. When the acute symptoms subside we reduce the dose to $2 \mathrm{cg}$. daily. In very severe cases one may employ the combination of ipecac, emetin and benzyl benzoate.

Pending diagnosis, it is our practice, in fulminating dysentery, to administer serum at once. Because of the acute and rapid course that severe bacillary dysentery runs we deem it to the best interests of the patient to give the serum liberally without waiting for the laboratory report. It can do no harm, and may do a great deal of good, for it is the experience of one of us (P. T. L.) that the serum treatment achieves its best results when applied early in the attack. We are cautious in the application of emetin in undetermined dysentery because of its mischievous effects on the heart that may be impaired in bacillary dysentery.

Microscopic Findings on Admission

\begin{tabular}{|c|c|c|c|c|c|c|}
\hline Case No. & $\begin{array}{l}\text { Endameoba } \\
\text { dysenterica }\end{array}$ & $\begin{array}{l}\text { Tricho- } \\
\text { monas } \\
\text { intestinalis }\end{array}$ & $\begin{array}{l}\text { Spirochaeta } \\
\text { eurygyrata }\end{array}$ & $\begin{array}{l}\text { Ankylo- } \\
\text { stoma } \\
\text { duodenale }\end{array}$ & $\begin{array}{l}\text { Trichuris } \\
\text { trichiura }\end{array}$ & $\underset{\text { dysenteriae }}{\text { Bacillus }}$ \\
\hline $\begin{array}{l}67452 \\
67528 \\
67798 \\
67888 \\
68117 \\
68143 \\
68374 \\
68213 \\
68529\end{array}$ & $\begin{array}{l}+ \\
+ \\
\ddot{+} \\
+ \\
+ \\
+ \\
+ \\
+\end{array}$ & $\begin{array}{l}\cdots \\
\cdots \\
\cdots \\
\cdots \\
\dot{+} \\
\because \\
+ \\
+\end{array}$ & $\begin{array}{l}\because \\
+ \\
\because \\
\therefore \\
\cdots \\
\cdots \\
\ddot{+}\end{array}$ & $\begin{array}{l}+ \\
\ddot{+} \\
\ddot{+} \\
+ \\
+ \\
\ddot{+}\end{array}$ & $\begin{array}{l}+ \\
\cdots \\
\dddot{t} \\
+ \\
+ \\
+ \\
+ \\
+ \\
+\end{array}$ & 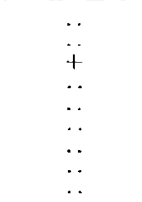 \\
\hline Total & 8 & 3 & 3 & $i$ & 7 & 1 \\
\hline
\end{tabular}

The benzyl benzoate we administered in a small amount of cold water three times a day after meals. The doses employed by us varied from 20 to 30 drops of the 20 per cent. alcoholic solution. It will be noted that Dr. Asuzano employed doses of 10 drops only. Notwithstanding this he seems to have secured results which, in the main, seem to be about as good as ours. In our next series we shall endeavor to determine the minimum effective dose as well as the effects of doses even higher than we have employed.

The protocols of our cases are as follows:

\section{REPORT OF CASES}

CASE 67452.-Male, 21 years of age, admitted to the hospital February 5. On admission the stool showed a scanty cellular exudate containing traces of mucus, erythrocytes and a few leukocytes. It was positive for Endameba dysenterica, Trichuris trichiura and ancylostoma duodenale.

The case presented an acute exacerbation of a chronic endamebiasis of about three months' standing, the original attack having occurred in Novem- 
ber, 1918. It lasted about one week. The present attack began three days before admission and was not at first accompanied by severe abdominal pain or tenesmus, but during the early part of his stay in the hospital the patient began to complain of both.

As this was not a case of marked severity, it was determined to treat it with benzyl benzoate alone. On the first night after treatment was started the patient slept well. The treatment was started on February 7, with a dose of 20 drops, three times a day. On the following day the abdominal pain and tenesmus were greatly diminished and the patient passed only two stools. The stools were becoming feculent. February 9, the pain and tenesmus had completely disappeared and the stools were practically normal in consistence. The patient was discharged free from dysenteric symptoms on February 16. His stools had averaged one a day since February 9.

This was undoubtedly a mild case. The stools were becoming formed on February 8, seven days after the onset of the attack. Under the benzyl benzoate treatment the patient showed almost immediate improvement which continued uninteruptedly to the day of his discharge.

CASE 67528.-Male, 35 years of age, admitted to the hospital February 8. The stool contained a rather abundant cellular exudate, blood and mucus. The stool was positive for Endameba dysenterica and Spirochacta eurygyrata. Bacteriologic examination was negative for Bacillus dysenteriae. Nevertheless, the character of the stool was such as to make us strongly suspect bacillary as well as entamebic involvement.

Clinically, the case presented a moderately severe type. The patient had just recovered from an attack of influenza complicated with bronchopneumonia. Six days before admission to the hospital he felt a growing sense of heaviness in the abdomen accompanied by tympanism. This lasted two days. During the following four days the bowel movements became more frequent (ten or more during the twenty-four hours). The movements were accompanied by tenesmus. They were mainly mucoid with a little blood.

On admission, the patient was having four to six movements during the day and from fifteen to twenty at night. The griping and tenesmus had become very severe. The colon was palpable and tender. The patient was very weak and unable to sleep. He was immediately given 20 c.c. of antidysenteric serum intramuscularly and was started on benzyl benzoate, 20 drops three times a day. The following day the bowel movemenis were diminished to four in the twenty-four hours. The night of February 10, the patient slept well. The pain and tenesmus were greatly diminished and the patient passed but two stools. February 11, there was a slight return of the abdominal pain and tenesmus, but both completely disappeared on the following day when the patient was able to be up and around the ward. The stools were well formed by this time and February 16 the patient left for home free from dysenteric symptoms.

It should be noted that this patient received only one dose of antidysenteric serum and that further treatment was carried out with benzyl benzoate alone. In view of this, it is questionable if the patient had a coexisting infection with Bacillus dysenteriae. The cellular exudate in the stool was, however, strongly suggestive of a concomitant bacterial infection of some kind.

Case 67888.-Male, 35 years of age, admitted to the hospital February 23. The stool contained blood, pus and mucus. There were numerous active Endamoeba dysenterica and Charcot-Leyden crystals. Other parasites present were Trichuris trichiura, and Spirochacta curygyrata. Bacteriologic examination for Bacillus dysenteriae was negative on two occasions. 
Notwithstanding the presence of pus we are convinced that this was a case of frank endamebiasis of a rather severe type. The patient had been suffering from the disease in a rather mild form, for about one month previous. On the day of admission the stools, which had been averaging about twenty in the twenty-four hours, became more frequent. They were scanty, mucoid and streaked with blood. There was marked tenesmus and abdominal pain. The patient was weak and unable to sleep at night.

Immediately after admission the patient was started on antidysenteric serum, 20 c.c. intramuscularly, every four hours. There was no improvement in his condition and February 25 he was started on 25 drops of benzyl benzoate, three times a day. The following day the patient passed only three stools as against nine the day before. There was a marked lessening of the abdominal pain and tenesmus and the patient said he felt much better in every way. The improvement continued, although on the following day the endamebae seemed more numerous in the stools. March 2, all pain and discomfort had disappeared and the stools were formed. March 5, there was complete absence of trophozoites and the concentrated stool failed to yield any cysts. The patient was discharged March 6.

CASE 68117.-Male, 55 years of age, was admitted to the hospital March 4. The stool contained much blood, but the cellular exudate was not abundant. Endanoeba dyscnterica was found as were Ancylostoma duodenale and Trichuris trichiura.

This case was of interest because of the complete absence of abdominal pain or tenesmus and we deemed it a favorable one on which to observe the effects of benzyl benzoate on peristalsis. The patient said he had suffered from dysentery for twenty-three years, so that the disease may be said to have been thoroughly established. The present attack started February 26, with profuse diarrhea, but with the discharge of neither blood nor mucus. Since the second day of the attack he had experienced difficulty in urination, the urine coming away drop by drop. This condition cleared up later.

On the day of admission the passing of stools was almost constant. The nights of March 5 and 6, ipecacuanha treatment was administered. March 6, benzyl benzoate was started, 30 drops, three times a day. That night the patient slept well for the first time since entering the hospital.

March 8, the general condition of the patient was greatly improved. The effect on peristalsis was very marked for the patient was almost constipated. Later, the stools fell to an average of one a day. The patient was feeling quite well March 11-so well that he insisted on leaving the hospital. His stool was still dysenteric and contained numerous active Endamoeba dysenterica. This patient promised to return for treatment on his next attack, which we assured him was probably not far distant.

CASE 68143.- Male, 17 years of age, was admitted to the hospital March 5. The stool contained little cellular exudate, but was laden with erythrocytes and Charcot-Leyden crystals. Endanoeba dysenterica was found. Other parasites present were Trichomonas intestinalis, Ancylostoma duodenale and Trichuris trichiura.

On admission the patient complained of abdominal pain and frequent bowel movements containing blood and mucus. The symptoms had developed eight days prior to admission. The patient was able to walk and did not seem particularly sick. There was no palpable mass in the abdomen, but there was slight tenderness on pressure over the ascending colon. The stools were mucoid and streaked with blood and the patient was passing them at the rate of ten to twelve in the twenty-four hours. Defecation was accompanied by pain and tenesmus. Pending diagnosis the patient had received 40 c.c. of antidysenteric serum intramuscularly on the night of admission.

March 7, the administration of benzyl benzoate, 30 drops, three times a day was started. There was little if any relief from the symptoms the following day, but the patient said he felt much better. March 9 he showed a distinct 
Improvement. The tenesmus had diminished in intensity and where the stools had the day before been passed at very frequent intervals, there were only twelve stools in the twenty-four hours. March 11, abdominal pain and tenesmus had disappeared completely and there were only three stools passed that day.

The stools had now become quite normal, and as there was no discomfort of any kind present, the benzyl benzoate was discontinued. Against our strongly expressed advice the patient insisted on leaving the hospital the following day. Unfortunately, his stool could not be obtained for examination before his departure. He was advised to return later for examination, but so far he has failed to put in an appearance. It may be said, however, that the last time his stool was examined microscopically (March 8) neither Endamoeba nor Trichomonas was found and the stool had lost most of its dysenteric character.

Treatment of this case was carried out wholly with benzyl benzoate, neither ipecacuanha nor its derivatives having been administered.

CASE 68374.-Female, 18 years of age, was admitted to the hospital March 15. The patient's stool contained much mucus and active trophozoites of Endamoeba dysenterica. Other parasites present were Ancylostoma duodenale and Trichuris trichiura.

This case presented a rather severe exacerbation of a chronic endamebiasis dating from 1915. The patient began to exhibit dysenteric symptoms with frequent bowel movements of a bloody and mucoid character, abdominal pain and tenesmus.

March 17, the patient was placed on benzyl benzoate (locally made), 20 drop's, three times a day. Two days later the pain and tenesmus had disappeared completely and on that day the patient's bowels moved only once. March 20, the patient failed to move her bowels, and as it was not desired to produce constipation, the benzyl benzoate was withdrawn and the patient was placed on a tonic of iron, quinin and strychnin and Fowler's solution of arsenic. $\mathrm{U}_{p}$ to the time of her discharge, April 14, no trophozoites or cysts of Endamoeba dysenterica could be found in the stools and the patient was gaining strength every day. She had shown no dysenteric symptoms since March 19. In other words, this patient received benzyl benzoate for three days only and yet apparently was completely cured of the attack. It should be noted in connection with this case that the patient received no anidysenteric treatment whatever, except the benzyl benzoate.

CASE 68529.-Male, 55 years of age, was admitted to the hospital March 21. Examination of the stool on admission showed the presence of active trophozoites of Endamoeba dysenterica, Trichomonas intestinalis and Spirochacta curygyrata and the ova of Ancylostoma duodenale and Trichuris trichiura. The helminthal infections were particularly heavy. By this, it will be seen that the patient was quite extensively parasitized, but such instances of multiple parasitism are not sufficiently rare in the Philippine Islands to excite comment when they do occur. The stool also contained a little pus and mucus.

This was an old chronic case with an acute exacerbation of great severity. The patient had been suffering intermittently from abdominal pains of a vague character since 1908 . He would have periodic attacks which lasted about two days and then subsided. It was apparently one of the fairly frequent instances where a chronic or subacute condition has dragged along for a period of years to finally reach a climax in a severe acute attack. The patient was asthenic and emaciated and although the colon was not palpable, there was marked tenderness at the hepatic flexure. The present attack started ten days previous to admission.

The patient was having very frequent bowel movements accompanied by much pain and tenesmus at the time of admission. He was put on ipecac and emetin. For four successive days he received $4 \mathrm{cg}$. of emetin hydrochlorid hypodermically and on three nights following he received $180 \mathrm{cg}$. of ipecac each night. The stools diminished in number, but were still passed frequently 
and on some days as many as fourteen in twenty-four hours. He gained somewhat in strength, but the pain and tenesmus were undiminished.

At this time the patient was started on the benzyl benzoate (locally made), 20 drops, three times a day. The following day he passed only two stools, but there was no lessening of the pain and tenesmus. The next day following, the pain and tenesmus disappeared completely and the patient had only one bowel movement. The improvement in his general condition was very marked. From that time on the patient never passed more than two stools during the twenty-four hours.

On the fourth day of the administration of the benzyl benzoate the patient began to complain of nausea and the drug was withdrawn with complete disappearance of the trouble. This is the only instance where we observed the slightest ill effects that could be attributed to the benzyl benzoate and it should be noted that the drug administered in his case had been locally prepared and was not the imported pharmaceutical preparation.

During the period when the patient was receiving ipecac and emetin, he was very sick and his condition was at one time extremely unfavorable in outlook. It was found necessary on several occasions to administer camphorated oil and hypodermoclysis. After the benzyl benzoate was withdrawn he received no further antidysenteric treatment until April 12, when a few cysts of Entamoeba dysenterica were found in his stool. He was placed on ipecac again for one night and the cysts were absent at the last examination of his stool before he was discharged, April 14. The stool, as in a previous case cited in this paper, was also free from Trichomonas but we attach no importance to this because these parasites frequently disappear from the feces for days at a time when the patient is undergoing no treatment whatever, only to reappear later. We doubt the efficacy of any drug which acts through the circulation against lumen dwelling parasites such as Trichomonas, unless the drug is eliminated in considerable volume into the lumen at the site of parasitism.

This case afforded an excellent basis for comparison between the effects of ipecacuanha and its alkaloids and benzyl benzoate, for the patient made no real substantial gain until the latter drug was started.

CASE 68213.-Male, 26 years of age, was admitted to the hospital March 9. The microscopic examination of the stool showed the presence of slight cellular exudate, erythrocytes and numerous Charcot-Leyden crystals. Numerous active trophozoites of Endamoeba dy'senterica were found and there were heavy infections with Trichomonas intestinalis and Trichuris trichiura. An interesting feature of the Trichomonas infection was furnished by the numerous agglomerations of these flagellates seen as the slide was studied.

The case was one of comparatively long standing, the patient complaining that he had suffered from dysenteric symptoms intermittently for the past two years. The present attack was of one month's duration and was accompanied by severe tenesmus.

March 11, the patient had thirteen bowel movements of the characteristic dysenteric type. The following night he was given one dose of 30 drops of the benzyl benzoate. Ten stools had been passed during that day, but March 13 the patient moved his bowels four times only. The supply of benzyl benzoate on the ward had given out and, througl a misunderstanding, was not renewed at that time. Ipecac treatment was started the night of March 14. That day the patient had moved his bowels five times, but there was no pain. However, he was very weak. The motions increased in frequence-possibly as a result of the laxative action of ipecac which occurs occasionally, but the increase continued together with a renewal of the tenesmus until March 17, when the patient had eleven movements. The ipecac was stopped and benzyl benzoate (of local preparation), 20 drops, three times a day, was started. There were three movements only on each of the succeeding days, pain and 
tenesmus had completely disappeared, but the stools were still dysenteric and contained many endamebae and trichomonads.

The benzyl benzoate was discontinued March 21, at which time the stool was practically formed and contained only slight streaks of blood. The withdrawal of the drug was accompanied in the next four days by a growing frequency in the number of the stools, a return of the pain and tenesmus and the reappearance of blood and mucus in the stool. The night of March 25, ipecac treatment was resumed. The stools gradually decreased in number, but it was not until March 30 that the patient experienced relief from pain and the stools had become formed. The patient was permitted to go home April 2 .

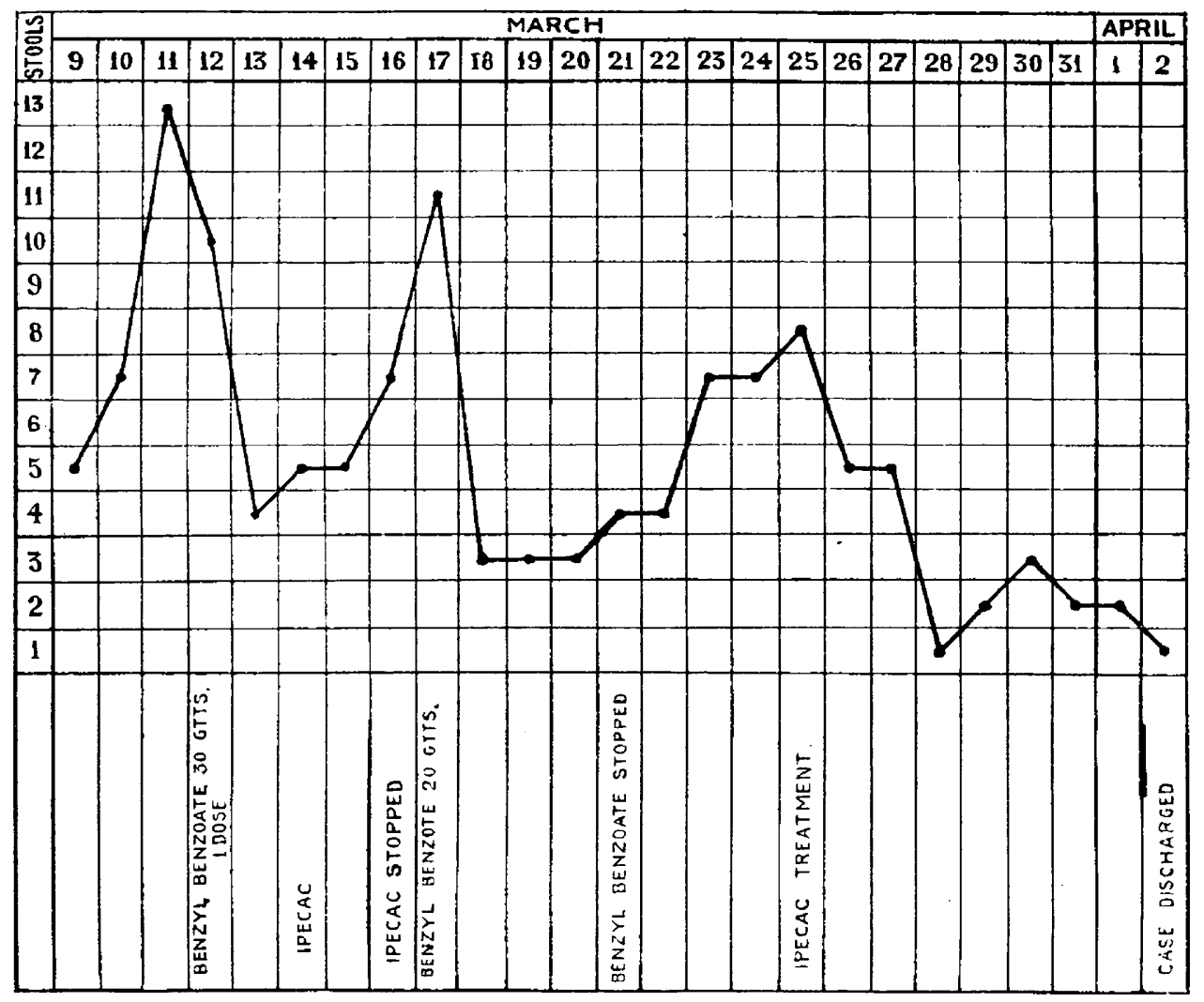

Chart of stools passed in Case 68213. Note sudden fall in number of stools following administration of one dose of benzyl benzoate March 12, and rise following administration of ipecac. Note also the sudden fall when benzyl benzoate was again given March 17, and the second rise when the drug was stopped March 21.

This case was one of the most interesting of the series for it seems to show, in a rather striking manner, the more or less positive response in the shape of the amelioration of symptoms each time benzyl is given. There is also a basis for comparison of the action of benzyl benzoate and ipecac. An attempt has been made to illustrate this graphically in the chart.

CASE 67798.-Male, 19 years of age, was admitted to the hospital February 19. The cytology of the stool presented the characteristic picture of 
bacillary dysentery, containing necrotic epithelium, polymorphonuclear leukocytes in abundance, blood and mucus. The only animal parasite found was Ancylostoma duodenale. Bacteriologically the stool was positive for Bacillus dysenteriae (Shiga).

This was a case of acute bacillary dysentery of the most severe type and at the outset we regarded the prognosis as exceedingly grave. The temperature at no time rose above $38.5 \mathrm{C}$, a condition we have grown to regard as rather an ominous sign in bacillary dysentery.

The onset of the attack was one week previous to admission to the hospital which did not mend matters for the patient. It started as diarrhea with black, foul-smelling stools, slight fever and chilly sensations. The stools became more and more frequent, gradually assuming a typical dysenteric character, then became more scanty in amount and, later, bloody. On admission the bowels were in almost continuous motion.

We approached the treatment of this case with benzyl benzoate with considerable hesitation. The indications for the administration of massive doses of antidysenteric serum were so apparent that treatment was started with 20 c.c. of the serum administered intramuscularly, every four hours. At the same time benzyl benzoate, 25 drops, three times a day, was given. The case ran a stormy course and it was not until February 25 that any change was noted. On that day the abdominal pain and tenesmus were markedly diminished and the patient stated that he felt much better. The following day the abdominal pain and tenesmus were still further relieved, the general condition showed distinct improvement and the stools a tendency to diminish in number. The serum was now administered only twice a day. The improvement continued and March 1 the temperature had fallen to normal, there was little pain or tenesmus and the stools were fewer in number though still numerous. The general condition of the patient was better.

From that time on the patient showed steady improvement. At times the temperature would be intermittent. March 8 , the stools were becoming formed and they were fully formed three days later. The patient was then averaging one to two movements in the twenty-four hours.

We are frank to say that we can make no quantitative estimate of the effect of benzyl benzoate in this case. Judged by our experience with similar cases, we feel that the drug helped. We believe that much of the general improvement that became manifest February 25 was due to the benzyl benzoate. Though we observed no bad effects, our inclination is still to employ this drug with considerable caution in the treatment of bacillary dysentery.

\section{DISCUSSION}

We have employed benzyl benzoate in the treatment of eight cases of endamebic dysentery uncomplicated by bacillary infection and have seen markedly good results in every case. All our cases were of the acute type and varied in severity. We have not as yet tried the action of the drug in chronic cases or in the cases of "carriers" or those persons who discharge abundant cysts without showing symptoms of endamebiasis.

We have, so far, observed no ill effects on the alimentary or excretory tracts following the administration of benzyl benzoate. In no case has the drug unfavorably altered the course of any case. On the contrary, as has been said, its administration has always been accompanied by a marked alleviation of both the objective and subjective symptoms of the disease. It gives the patient much needed rest and 
permits him to sleep at night, a most important desideratum in the treatment of dysentery.

Under the administration of the drug the endamebas disappeared from the stools in nearly every case as the general symptoms subsided. Whether this disappearance of the parasites is temporary or permanent remains to be demonstrated by future investigation.

There is ground for belief that benzyl benzoate possesses amebicidal properties. This has been suggested by Macht and Fisher ${ }^{6}$ in a series of in vitro experiments. While we do not place a high value on such experiments, we consider the point is well worth working on. We can only say that this series of cases does not afford evidence which would tend to establish proof one way or the other.

We believe that in addition to relieving, to a substantial degree, some of the most distressing symptoms which accompany a dysentery attack that, in combination with ipecacuanha or its alkaloids, benzyl benzoate noticeably shortens the term of illness.

It has been shown in some of our cases that benzyl benzoate alone will bring about a complete subsidence of the symptoms of acute endamebic dysentery. Whether these cases are permanently cured or will be subject to relapse must be determined in the future. It is well known that emetin cannot be relied on to cure all cases of chronic endamebiasis and the action of benzyl benzoate adds another factor to the problem of the mechanism of the cure of endamebiasis.

There is certain evidence that when used with care. benzyl benzoate may be employed with advantage in conjunction with serum, in the treatment of bacillary dysentery. It is suggested that it may be of particular value in mixed bacillary and endamebic dysentery where the condition of the heart forbids the use of emetin.

For the present, we consider it safe to state that benzyl benzoate is, to say the very least, a valuable auxiliary to the other forms of antiamebic treatment, and that it gives real promise of usefulness when employed alone. This is entirely aside from any amebicidal powers it may, in the future, be shown to possess. It is agreeable to take, and if it can be used alone it will be a boon to the patient who suffers from the distressing nausea produced by ipecac.

We believe it is probably unwise in acute cases, especially when a bacillary involvement is present with accompanying toxemia, to push the drug to the point where the patient becomes constipated - a condition that has occurred in two of our cases of uncomplicated endamebiasis.

6. Macht, David I., and Fisher, E.: J. Pharmacol. \& Exper. Therap. 11: 263 (April) 1918. 
Benzyl benzoate seems to take the place of morphin in dysentery in that it slows the peristalsis and relieves the pain and tenesmus; but it shows none of the undesirable traits of opium. It is slower in its effects than opium and, of course, obviates the danger of habit formation in a susceptible patient. The sedative action of the drug on the intestinal musculature seems to us to be of preeminent importance. The violent peristalsis is promptly quieted and the intestine is given rest and a chance to carry out the normal reparative processes. It is possible that the spread of the infection is also distinctly limited through the quieting of the gut. Rest for the intestine seems to us to be fully as important as bodily rest for the patient secured by keeping him in bed.

So far as we have observed, benzyl benzoate exerts no effects on any of the intestinal protozoa other than Endameba dysenterica, neither were the intestinal helminths encountered affected by it.

\section{CONCLUSIONS}

It seems evident that benzyl benzoate exerts a definite and favorable influence on the course of endamebic dysentery. The question arises as to how this effect is brought about. Among the points that should be determined in the future are the following:

1. Its action on chronic and "carrier" cases and in hepatic endamebiasis as well as endamebic involvement of the urinary system and. other organs.

2. The determination of the minimum effective dose, and the action of larger doses than we have given.

3. Its effects on the hemopoietic system.

4. Has the drug specific amebicidal properties, possibly through the splitting off of one or more of its radicals in metabolism? If such is the case a curative action may be expected.

5 . Is the effect more on the host than on the parasite? That is to say, does it have a tendency to restore the normal equilibrium between parasite and host with accompanying subsidence of the acute symptoms and assumption of a state of chronicity, but without sterilization of the patient? Under such circumstances relapse to an acute condition may be expected sooner or later.

6. Does the sedative action of the drug on the intestinal musculature merely limit the spread of the infection and possibly permit some undetermined body defense to regulate the infection?

7. Is there a possibility of synergistic action between benzyl benzoate and ipecacuanha and its alkaloids, or, is it possible that benzyl benzoate tends to make the endameba more sensitive to the action 
of ipecac and emetin? In this connection attention is drawn to Case 68313.

In conclusion, we desire to extend our thanks to Dr. Ariston Bautista y Lim, head of the department of medicine on whose service these patients were assigned, for permission to study them, and to Drs. Facundo Esquivel, F. R. Fernandez de Leon and Wenceslao Vitug of the house staff for substantial assistance rendered.

\title{
ADDENDUM
}

\section{TWO CASES OF ENDAMEBIC DYSENTERY AT BAGUIO HOSPITAL TREATED WITH BENZYL BENZOATE}

\author{
M. A. ASUZANO, M.D. \\ BAGUIO
}

These two cases of acute endamebic dysentery were treated by me at the Baguio Hospital at the instance of Professor Haughwout, who furnished me with a supply of benzyl benzoate miscible in 20 per cent. alcoholic solution. They are of some interest inasmuch as one of the patients received emetin compounds in addition to the benzyl benzoate, while the other was treated with the benzyl benzoate alone.

The following is a report of my cases:

\section{REPORT OF CASES}

CASE 1.-C. Y., a Spanish mestiza, 31 years of age, had dysentery six or seven months ago at which time her stool was reported positive for Endamoeba dysenterica. Two physicians treated her at that time by both hypodermic and intravenous administration of emetin hydrochlorid. She suffered three relapses. The patient came to Baguio and two days later came down with another acute attack. Her stool contained an abundance of mucus and blood and a large number of actively motile endamebas.

I gave her six injections of emetin, in one-half grain doses, and, she having failed to improve, gave her in all, six grains of emetin bismuthous iodid. Examination of the stool following this treatment, showed numerous cysts of Endamoeba dysenterica, but three days later she came down with another acute attack. I continued the treatment with emetin bismuthous iodid, giving her 12 grains in all. About a week after the discontinuance of the drug, she had another acute attack, and, as in the previous instance, had from ten to fourteen bowel movements a day. These were accompanied by intense griping pains in the abdomen, intense burning pain in the region of the rectum, accompanied by distressing tenesmus.

At this period I started her on the benzyl benzoate, 10 drops three times a day. After taking three doses of the benzyl benzoate the number of bowel movements fell to four. The stools still contained mucus, slightly streaked with blood and endamebas. The abdominal pain and distress were by this time markedly diminished. The second day the patient had but one movement of a semisolid nature. Pain in the abdomen had disappeared almost altogether as had the pain in the rectum.

However, the stools still bore traces of mucus and contained few endamebas. so $I$ put her on emetin again, giving her, in all, 2 grains of the hydrochlorid and 6 grains of the emetin bismuthous iodid. 
At the end of a week two stool examinations failed to show the presence of blood, mucus or endamebas. Two examinations by the concentration method of Cropper and Row failed to show the presence of cysts.

CASE 2.-J. S., Filipina, 24 years of age, was already under treatment in the hospital for pulmonary tuberculosis. She was in the second stage of that disease. She developed endamebic dysentery, her stool showing an abundance of mucus, blood and active Endamoeba dysenterica. She was passing eight to ten stools a day. These were accompanied by distressing gripping pains in the abdomen, burning pain in the rectum and tenesmus. On the second day of the illness $I$ started her on 10 drops of the benzyl benzoate, three times a day. Following the first three doses the daily number of stools fell to two. These were of semi-solid character, but still contained mucus, blood and endamebas. The parasites were very sluggish in their movements. No cysts were found. The pain was much diminished in intensity, but had not disappeared. On the second, third and succeeding days the bowel movements were normal and no endamebas were found either as trophozoites or encysted forms. All pain and distress had disappeared.

\section{COMMENT}

The first case was the more severe of the two. Treatment included the administration of emetin bismuthous iodid, and later emetin hydrochlorid, but under it little, if any, improvement was noted in the symptoms. Later, emetin hydrochlorid was combined with benzyl benzoate which brought about a complete and speedy subsidence of all the symptoms and the disappearance of the parasites from the stool.

No emetin was given to the second patient. Under treatment with benzyl benzoate the symptoms rapidly disappeared and, with them, the endamebas in both the trophozoite and encysted forms disappeared. 